\title{
Fennel main constituent, trans-anethole treatment against LPS-induced acute lung injury by regulation of Th17/Treg function
}

\author{
SICHAO ZHANG $^{1 *}$, XI CHEN $^{1 *}$, ICHINKHORLOO DEVSHILT $^{1 *}$, QI YUN $^{2}$, \\ CONG HUANG $^{1,3}$, LIJUN AN ${ }^{1}$, SOSORBURAM DORJBAT ${ }^{1}$ and XIN HE ${ }^{1}$ \\ ${ }^{1}$ School of Chinese Materia Medica, Tianjin University Traditional Chinese Medicine, Tianjin 300193; \\ ${ }^{2}$ Xinjiang Tefeng Pharmaceutical Co., Ltd., Urumqi, Xinjiang 830054; ${ }^{3}$ School of Basic Medicine, \\ Guiyang College Traditional Chinese Medicine, School of Basic Medicine, Guiyang, Guizhou 550002, P.R. China
}

Received October 30, 2017; Accepted April 25, 2018

DOI: $10.3892 / \mathrm{mmr} .2018 .9149$

\begin{abstract}
Fennel, commonly used in traditional Chinese medicine, is known as Foeniculum vulgare Mill. And its clinical application has been shown to target many biological systems including gastroenterology, endocrinology, gynecology and respiratory. The main constituent of the fennel essential oil is trans-anethole, which has been described to have anti-inflammatory and antibacterial activities. The aim of the present study was to define the anti-inflammatory influence in acute lung injury (ALI)-bearing mice. For 3 days, ALI-bearing mice were induced by lipopolysaccharide (LPS) suspension in normal saline $(24 \mathrm{mg} / \mathrm{kg})$. On the fourth day, the trans-anethole was administrated $(36.4,72.8$ or $145.6 \mathrm{mg} / \mathrm{kg}$ ) as well as dexamethasone $(5 \mathrm{mg} / \mathrm{kg})$ once per day for 7 consecutive days in mice. Following the completion of drug administration mice were sacrificed. Hematoxylin and eosin staining was performed in the lung paraffin section, for comparisons between monocyte and eosinophil cells in bronchoalveolar lavage fluid. The relative gene expression of interleukin (IL)-10 and IL-17 was determined by reverse transcription-quantitative polymerase chain reaction. These two cytokines and the proportion of T helper 17 (Th17) cells and $\mathrm{T}$ regulatory (Treg) cells were determined by flow cytometry. The main constituent of fennel, trans-anethole, eliminated LPS-induced histopathological changes, decreased the number of inflammatory cells and resulted in a notable reduction in IL-17 mRNA expression. In addition, trans-anethole increased
\end{abstract}

Correspondence to: $\mathrm{Dr} \mathrm{Xin} \mathrm{He}$, School of Chinese Materia Medica, Tianjin University Traditional Chinese Medicine, 312 Anshanxi Road, Tianjin 300193, P.R. China

E-mail: hexintn@163.com

${ }^{*}$ Contributed equally

Key words: fennel, acute lung injury, trans-anethole, T regulatory cells, T helper cell 17
IL-10 mRNA expression in isolated lung tissues and resulted in a marked elevation in Treg cells and reduction in Th17 cells in spleen tissues. The results of the present study indicated that the main constituent of fennel, trans-anethole may be an anti-inflammation component, which influenced the regulation of Th17/Treg responses. Therefore, this medicinal herb may support a healing effect on diseases of inflammatory.

\section{Introduction}

Foeniculum vulgare is from the Umbelliferae family of plants and is used as an antiaging agent to promote longevity. It is more commonly called fennel and widely used as a medicine especially in Asia, but also in other places worldwide $(1,2)$. Traditional practices and the scientific assessment of fennel indicate that it is a widely applied herbal plant in human health care in many developing and developed countries (3). In fact, it has been attested to have a number of therapeutic properties for the treatment of internal organs, especially in the endocrine, gastrointestinal tract and respiratory systems (4-6). In addition, fennel is widely used for the treatment of inflammatory diseases in conventional medicine (7).

The volatile chemical composition of wild fennel samples harvested in different geographical areas has been widely reported (8). Trans-anethole [1-Methoxy-4-(E)-propenyl-ben zene] is an organic compound isolated from fennel (9). The uses of fennel have been evaluated in a number of biopharmacological studies that suggest that the various constituents of fennel (seeds, leaves and fruits) are rich in molecules with antioxidant activity (10-12). Furthermore, the extracts and components of fennel have been reported to have anti-inflammatory, anti-bacterial and antiviral effects (13-15).

In 1967, acute lung injury (ALI) was defined as mild acute respiratory distress syndrome (ARDS), with a $\mathrm{P} / \mathrm{F}$ ratio of $<300 \mathrm{mmHg}(40 \mathrm{kPa})$ and positive end-expiratory pressure/continuous positive airway pressure levels of $>5 \mathrm{~cm}$ $\mathrm{H}_{2} \mathrm{O}$, according to the Berlin Definition (16). Classical signs of ALI include accumulation of neutrophils, increased alveolar fluid and pro-inflammatory cytokines in the lungs (17). Worldwide, $5 \%$ of the population succumb to ALI per year. 
This syndrome involves acute inflammation of the bronchi in the lungs. The clinical manifestations of ALI are a productive cough, wheezing, shortness of breath, fever and chest distention. Regeneration of the alveolar epithelium after ALI is important for maintaining homeostasis, and the latest studies demonstrate that the immune system may influence the protection of epithelial surfaces by increasing barrier function and supporting healing $(18,19)$.

A recent study demonstrated that $\mathrm{CD}^{+} \mathrm{T}$ lymphocytes, particularly $\mathrm{T}$ helper 17 (Th17) cells and regulatory $\mathrm{T}$ cells (Tregs) are an essential subject for research into the etiology or pathogenesis of ARDS. Th17 cells, a recently identified active subdivision of $\mathrm{CD}^{+} \mathrm{T}$ cells and the main origin of interleukin (IL)-17 (IL-17A), play a major role in protecting the body from microorganisms, such as Staphylococcus aureus $(20,21)$. Patients who suffer from ALI/ARDS have increased levels of T-cell proliferation and activation, especially Th17 cells (22). Tregs act in an anti-inflammatory role primarily by contact-dependent destruction of immunocytes or release of cytokines like IL-10 (23). A reduction in the proportion of Treg cells or a decrease in Treg cell function is associated with a number of autoimmune disorders (24). Tregs alter innate immune reactions during lung injury and may play a role in treating ALI as indicated by Guo et al (25). Mature Tregs can be classified as inducible Tregs (iTregs) or natural Tregs (nTregs). iTregs are able to perform their inhibitory function by producing immunosuppressive cytokines like IL-10 (26). Tregs differ from Th17 cells and have a powerful pro-inflammatory action by generating IL-17. In pre-clinical and clinical trials on pulmonary and autoimmune diseases, Th17 cells have been reported to play a role (27-30). The IL-17 from Th17 cells inhibits Treg differentiation $(31,32)$. Additionally, IL-17A could be a pro-inflammatory cytokine and may play a key role in ALI induced by lipopolysaccharide (LPS), as shown by previous studies $(33,34)$. In addition, lung injury by Bordetella pertussis is promoted by increased levels of Th17 cells (35). However, the main mechanism of CD4 ${ }^{+}$ $\mathrm{T}$ cells in ALI requires further investigation. These studies have revealed that mice with LPS-induced lung injury may be treated by both Cozaar ${ }^{\mathrm{TM}}$ and alanylglutamine, which act by decreasing the Th17 cell immune responses and affecting the Th17/Treg balance in favor of Treg cells (36). The pathological mechanism of autoimmune and inflammatory disease involves a balance between Th17 and Tregs. A Treg/Th17 imbalance is associated with the occurrence of autoimmune diseases. Further investigation into these cell populations in airway inflammation may improve the understanding of the immune mechanism of lung disease.

In the present study, we first established a model of LPS-induced ALI and then treated the mice with fennel volatile oil. The volatile oil reduced exudation in the lungs and also significantly reduced the quantity of leukocytes infiltrating into bronchoalveolar lavage fluid (BALF). The above results showed that the volatile oil of fennel could inhibit ALI in mice.

As a result of the comprehensive analysis of the function of the fennel active constituent trans-anethole in the literature, we know that it has anti-inflammatory, insecticidal activity and estrogen activity (37). In our study we investigated the effect of trans-anethole on lung inflammation in ALI, as well as the related modalities for the function of Th17 and Tregs.

\section{Materials and methods}

Animals and cell culture. According to the guidelines of the Tianjin University of Traditional Chinese Medicine (Tianjin, China) and the Animal Ethics Committee of Laboratory Animal Care, all animal studies received ethical approval. Sixty healthy 5-week-old male C57BL/6 mice (18-22 g) were ordered from the Animal Research Center of the Chinese Academy of Medical Sciences (Beijing, China) and were housed under suitable conditions. Mice were raised on standard water and food.

Drugs and reagents. LPS, dexamethasone and trans-anethole were purchased from Sigma-Aldrich (Merck KGaA, Darmstadt, Germany). Oligo DNA was purchased from Shanghai Bio-engineering Co. (Shanghai, China). TransScript First-Strand cDNA Synthesis SuperMix was obtained from TransGen Biotech (Beijing, China). SuperReal PreMix Plus was obtained from Tiangen (Beijing, China). PE-labeled anti-mouse IL-17 and IL-10, fluorescein isothiocyanate (FITC)-labeled anti-mouse CD4, Alexa Fluor ${ }^{\circledR}$ 647-labeled anti-mouse Foxp3, anti-mouse M1/70+ and anti-mouse F4180+ were purchased from Santa Cruz Biotechnology, Inc. (Dallas, TX, USA). The PE anti-mouse Foxp3 Staining Set was from eBioscience (Thermo Fisher Scientific, Inc., Waltham, MA, USA).

Animal model and experimental procedures. These sixty mice were allowed to adapt to normal laboratory conditions during the experiment. The experimental groups (10 mice/group) were as follows: normal control, LPS-induced model, trans-anethole treatment (low: $36.4 \mathrm{mg} / \mathrm{kg}$; medium: $72.8 \mathrm{mg} / \mathrm{kg}$; and high: $145.6 \mathrm{mg} / \mathrm{kg}$ ) and dexamethasone treatment $(5 \mathrm{mg} / \mathrm{kg})$. As presented in Fig. 1, the pulmonary delivery model was established in normal mice. The C57BL/6 mice bronchitis model was induced by intratracheal instillation of LPS suspension in normal saline $(24 \mathrm{mg} / \mathrm{kg}$ ) for 3 days. On the 4th day, the mice were administered trans-anethole $(36.4,72.8$ or $145.6 \mathrm{mg} / \mathrm{kg}$ ) and dexamethasone $(5 \mathrm{mg} \mathrm{kg} / \mathrm{kg}$ ) once a day for 7 consecutive days. The mice were sacrificed $24 \mathrm{~h}$ after the end of treatment.

Histopathology analysis. The lung tissue was stained with hematoxylin and eosin and the inflammatory changes and morphological lesions were analyzed using a light microscope (magnification, $\mathrm{x} 400$ ).

Inflammatory cell count in BALF. A total of $50 \mu \mathrm{l} / \mathrm{g}$ sterile saline was lavaged twice, then the BALF was collected and centrifuged at 3,000 x g for $15 \mathrm{~min}$. The white blood cells in the BALF were divided and counted into two types, containing monocytes and eosinophils.

Reverse transcription-quantitative polymerase chain reaction (RT-qPCR) analysis in spleen and lung tissue. Total RNA from the isolated spleen and lung tissues was reverse transcribed into first strand cDNA, according to the manufacturer's instructions. The relative mRNA levels of samples were measured using a NanoDrop 2000. qPCR was performed with sequence-specific primer pairs to execute a 40-cycle two-step PCR procedure. The qPCR thermocycling conditions were 


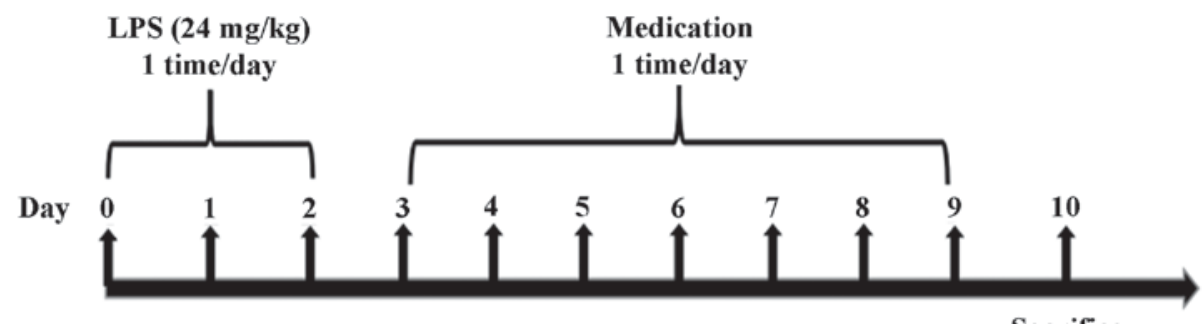

Sacrifice

Figure 1. Study methods for establishing the model in mice with acute lung injury and the administration of medication. Bronchitis model was induced in mice by LPS suspension in normal saline ( $24 \mathrm{mg} / \mathrm{kg}$ ) on days 0 to 2 . The mice were administrated $2 \mu \mathrm{l} / \mathrm{g}$ LPS for 3 days. On the fourth day, different doses of trans-anethole and dexamethasone were orally administrated for 7 consecutive days. The model group was only given normal saline administration. The control group was not given anything. Following the completion of drug administration, the mice were subsequently sacrificed within $24 \mathrm{~h}$ post-treatment. LPS, liposaccharide.

as follows: $95^{\circ} \mathrm{C}$ for $3 \mathrm{~min}, 40$ cycles of $95^{\circ} \mathrm{C}$ for $15 \mathrm{sec}$ and $60^{\circ} \mathrm{C}$ for $30 \mathrm{sec}$, then $95^{\circ} \mathrm{C}$ for $30 \mathrm{sec}$. Primers were designed by Primer Premier 3.0 (Premier, Vancouver, BC, Canada) and were as follows: IL17, forward, 5'-CCTCAAAGCTCA GCGTGTCC-3' and reverse, 5'-GAGCTCACTTTTGCGCCA AG-3'; IL-10, forward, 5'-AAGTGATGCCCCAGGCA-3' and reverse, 5'-TCTCACCCAGGGAATTCAAA-3'; GAPDH, forward, 5'-AACGGATTTGGCCGTATTGG-3' and reverse, 5'-CCTTGACTGTGCCGTTGAAT-3'. Gene expression of IL-17 and IL-10 was measured with qPCR using SuperReal PreMix Plus (Tiangen). The fold-change of every gene was quantified by the $2^{-\Delta \Delta \mathrm{Cq}}$ method and the internal control was GAPDH gene. The data were normalized to the internal reference gene.

Th17/Treg examination in the splenic tissue. Flow cytometry instrument (BD Biosciences, Franklin Lakes, NJ, USA) was used to measure Th17 and Tregs in the splenic tissue. As described in the manufacturer's protocol, the spleen cells were stained with the FITC anti-mouse CD4 antibody, then fixed and permeabilized with PE anti-mouse Foxp3 Staining Set. Then, the cells were stained with anti-mouse IL-10 and anti-mouse IL-17 (eBioscience; Thermo Fisher Scientific, Inc.).

IL-17 and IL-10 cytokine expression in $C D 4^{+} T$ cells isolated from the spleen of WT mice. The spleen cells were stained using anti-CD4 antibodies, incubated and fixed with IL-10 and IL-17 staining sets. According to the manufacturer's instructions, the cells were stained with PE-labeled anti-mouse IL-17 and IL-10 and then tested by flow cytometry (BD Biosciences).

Statistical analysis. All data are presented as the mean \pm standard deviation of the mean and compared by one-way analysis of variance followed by Tukey's post hoc test. All data were analyzed by SPSS 19 (SPSS, Inc., Chicago, IL, USA). P<0.05 was considered to indicate a statistically significant difference.

\section{Results}

Trans-anethole attenuates airway inflammation in ALI mice. Lung tissues were collected in each group. From analyzing the histological results, the lung sections of the normal group exhibited normal architecture and there was no obvious neutrophils infiltration, erythrocytes or protein exudate (Fig. 2A). However, severe protein exudation and lung cell infiltration were demonstrated in the ALI mouse model (Fig. 2B). These were relieved after trans-anethole and dexamethasone treatments.

Trans-anethole decreases the number of inflammatory cells in the BALF of ALI mice. The number of inflammatory cells was measured in the BALF. An increase in the level of leukocytes was caused by LPS with both eosinophilic granulocytes and mononuclear cells being enhanced compared with the normal group. Compared with model group, Trans-anethole treatment significantly decreased the level of inflammation cells in the BALF. Compared with dexamethasone, the numbers of eosinophils and monocytes were not significantly different in the BALF compared with the trans-anethole group. The number of monocytes was reduced in the high group compared with the other groups. As expected, the level of inflammatory cells was markedly decreased in BALF following trans-anethole treatment (Fig. 3).

Trans-anethole regulates cytokine $m R N A$ expression in isolated spleen and lung tissues of ALI mice. Both anti-inflammatory cytokine IL-10 and pro-inflammatory cytokine IL-17 are critical for inflammation. Tregs can decrease inflammation via the production of immunosuppressive agents such as IL-10. To identify the role of trans-anethole on IL-17 and IL-10 expression, we measured the expression of both cytokines in the lung and splenic tissue of mice in each group.

As shown in Fig. 4, IL-17 was markedly elevated in the model group compared with the normal group in the spleen and lung tissue. The level of IL-17 was significantly reduced in the trans-anethole group compared with the model groups. Compared with dexamethasone, the level of IL-17 was decreased in the trans-anethole group. The level of IL-17 in spleen and lung tissue was significantly reduced in the trans-anethole medium group compared with the other groups.

As shown in Fig. 5, the level of IL-10 was not changed in the model group compared with the normal group in the spleen and lung tissue. Although compared with the dexamethasone group, the level of IL-10 was slightly decreased in the spleen and lung tissue in the trans-anethole group, trans-anethole treatment dramatically increased IL-10 expression compared with the model group in the spleen and lung tissue. These results showed that trans-anethole can decrease IL-17 and increase IL-10 in ALI mice, which demonstrates the effectiveness of the treatment on lung inflammation. 

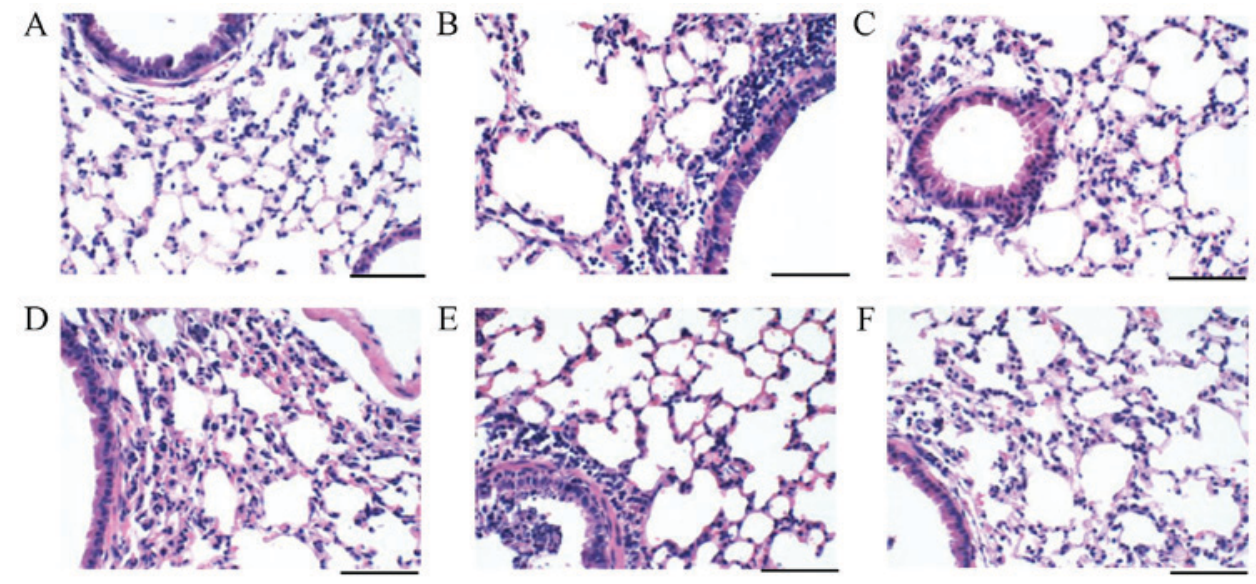

Figure 2. Trans-anethole alleviated liposaccharide histopathological illustration in lung tissues of mice. Lung tissue of the (A) Normal, (B) Model, (C) Dexamethasone, (D) Low (E) Medium and (F) High groups were exposed to histopathological illustration by hematoxylin and eosin staining (scale bars, $100 \mu \mathrm{m}$; magnification, $\mathrm{x} 400)$.
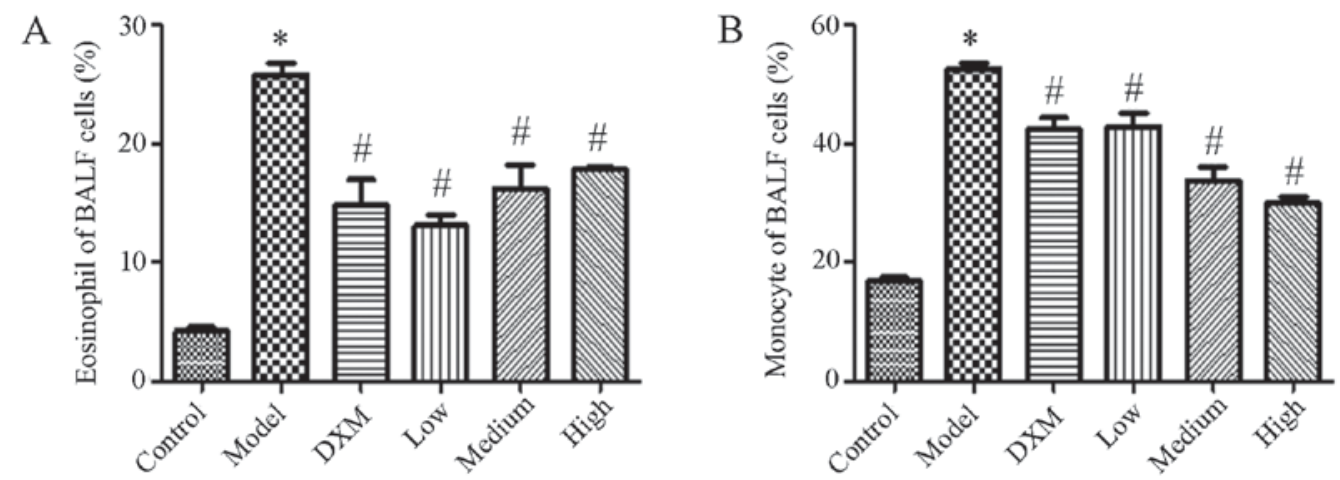

Figure 3. Trans-anethole treatment inhibits (A) eosinophil and (B) monocyte cell level in BALF in mice. Statistics were displayed as the mean \pm standard deviation ( $\mathrm{n}=5$ ). ${ }^{\text {P }}<0.05$ vs. control; ${ }^{*} \mathrm{P}<0.05$ vs. model. BALF, bronchoalveolar lavage fluid; DXM, Dexamethasone.

A

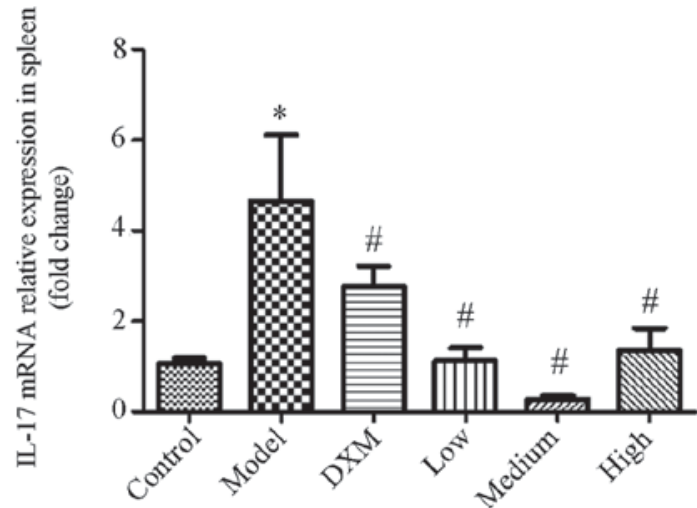

B

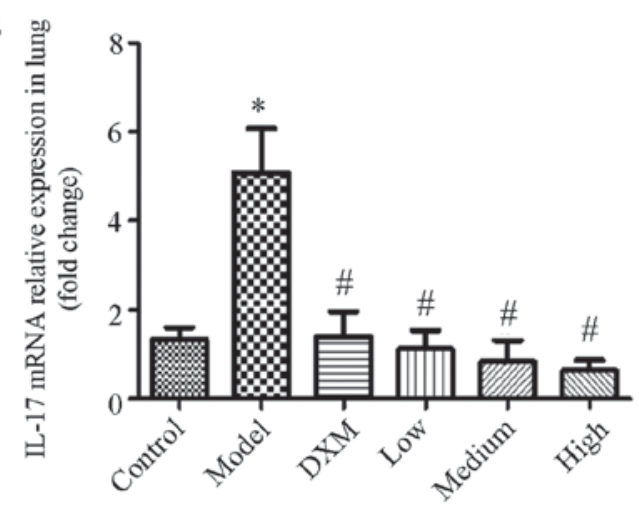

Figure 4. Trans-anethole regulates IL-17 mRNA relative expression in spleen tissue in mice. IL-17 mRNA relative expression in (A) the spleen and (B) the lungs. Statistics were showed as the mean \pm standard deviation $(n=6)$. ${ }^{*} \mathrm{P}<0.05$ vs. control; ${ }^{\#} \mathrm{P}<0.05$ vs. model. IL, interleukin; DXM, Dexamethasone.

Trans-anethole controls the proportions of Th17 and Treg in the spleen of ALI mice. Given the important role of IL-10 and IL-17 for Th17 differentiation, we aimed to analyze the effect of trans-anethole on these two cytokines. $\mathrm{CD} 4^{+} \mathrm{T}$ cells were collected from the spleen of mice in the absence or presence of trans-anethole. We observed that the compound selectively inhibited IL-17 production without affecting the secretion of IL-10 (Fig. 6).
To assess the proportion of Tregs and Th17 cells that were involved in lung inflammation, changes in the ratio of the two groups of cells in the mice spleens was measured using flow cytometry. As shown in Fig. 7, an obvious increase in Th17 cells was demonstrated in the model group compared with the normal group in the spleen. Trans-anethole treatment caused a significant decrease of the proportion of Th17 cells and a significant increase in the percentage of iTreg cells 
A

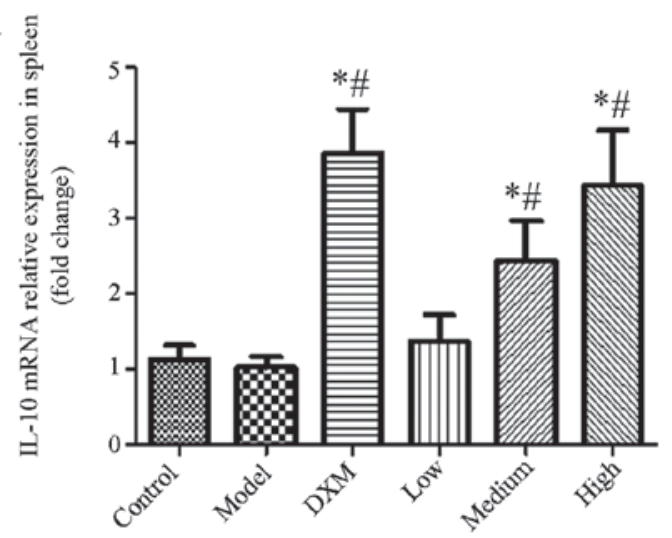

B

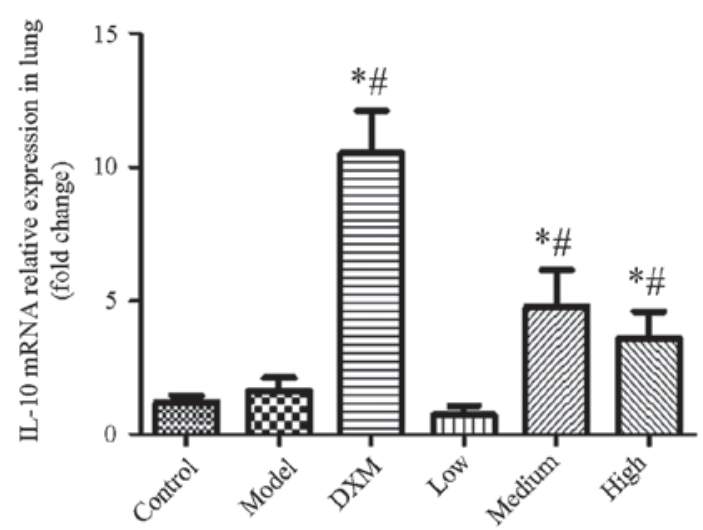

Figure 5. Trans-anethole regulates IL-10 mRNA relative expression in mouse lung tissue. IL-10 mRNA relative expression in (A) the spleen and (B) the lungs. Statistics were displayed as the mean \pm standard deviation $(n=6) . ~ * P<0.05$ vs. control; ${ }^{*} \mathrm{P}<0.05$ vs. model. IL, interleukin; DXM, Dexamethasone.
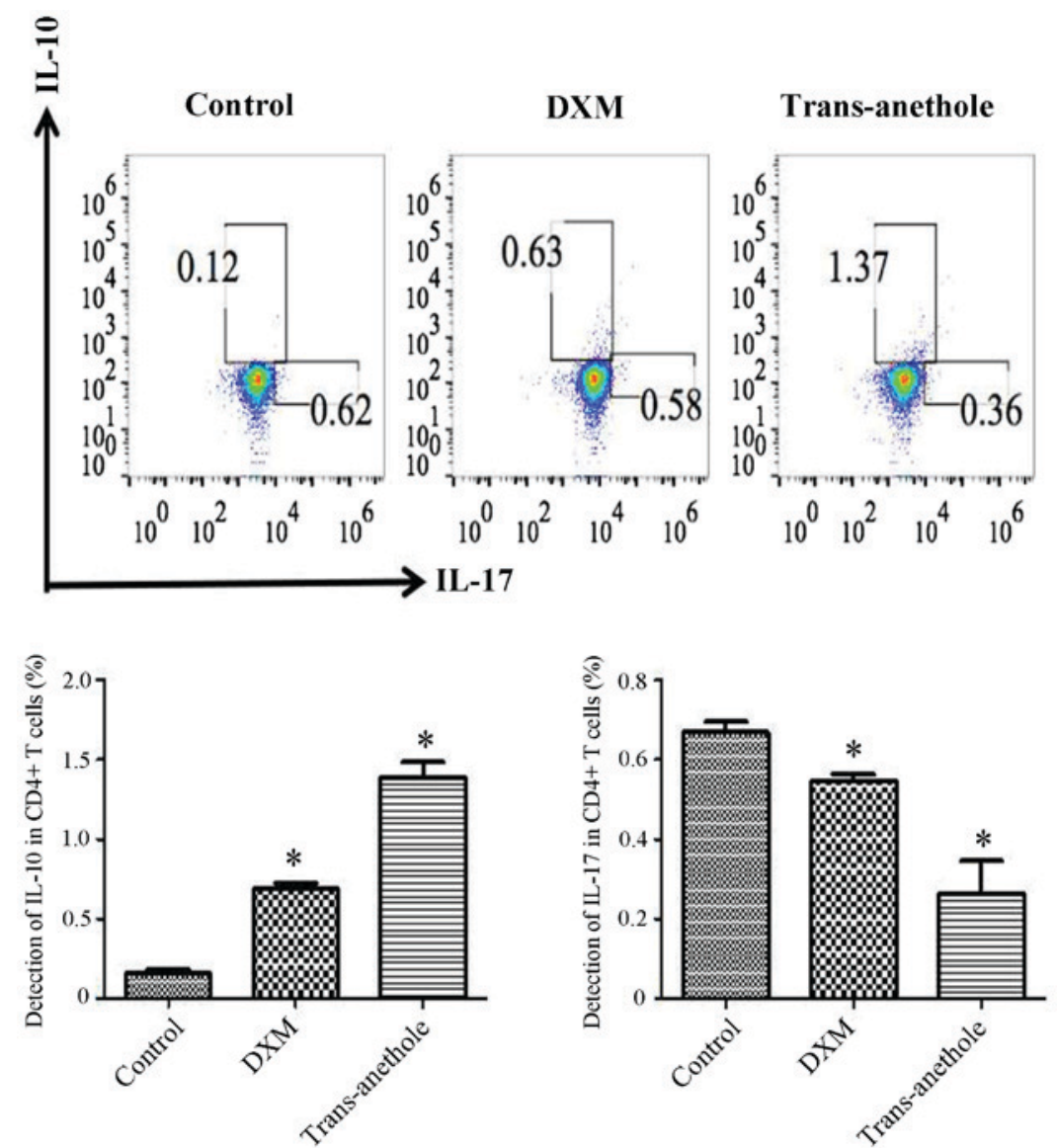

Figure 6. Trans-anethole regulates IL-10 and IL-17 cytokine expression in CD4 ${ }^{+} \mathrm{T}$ cells isolated from the splenocytes of wild-type mice. Statistics were displayed as the mean \pm standard deviation $(n=6)$. ${ }^{*} \mathrm{P}<0.05$ vs. control. IL, interleukin; DXM, Dexamethasone.

compared with the LPS-induced ALI mice. Compared with the dexamethasone-treatment group, the changes to the ratio of Th17 and Treg cells were more obvious in the spleen in the trans-anethole group.

\section{Discussion}

LPS is a stimulator of pro-inflammatory cells, therefore intratracheal administration of LPS was used to establish the ALI mouse model. Histological examination of the lung tissue showed thinning alveolar walls, diffuse hemorrhage, interstitial tissue inflammation and decrease of the size of the alveolar cavity. Our results also showed that inflammatory cells (eosinophils and monocytes) were reduced in the BALF after treatment.

ALI is a common disease. The early stages of inflammatory diseases start with increasing levels of inflammatory cells and mediators The CD $4^{+}$Foxp $3^{+} \mathrm{T}$ cell Treg subdivision is important for influencing two parts of the immune process (1): To subdue an extensive range of effector immune 
A Control

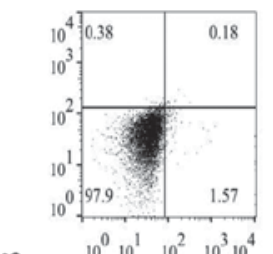

产

High

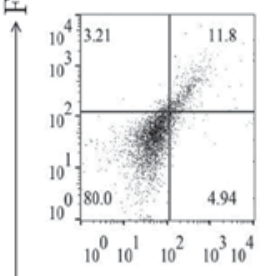

Model

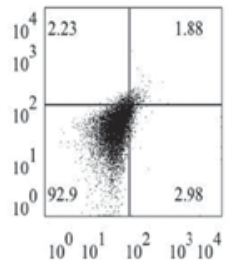

Medium

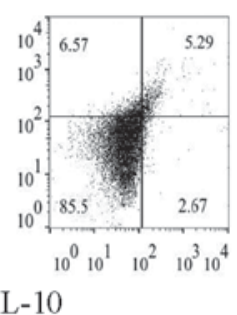

Model

B

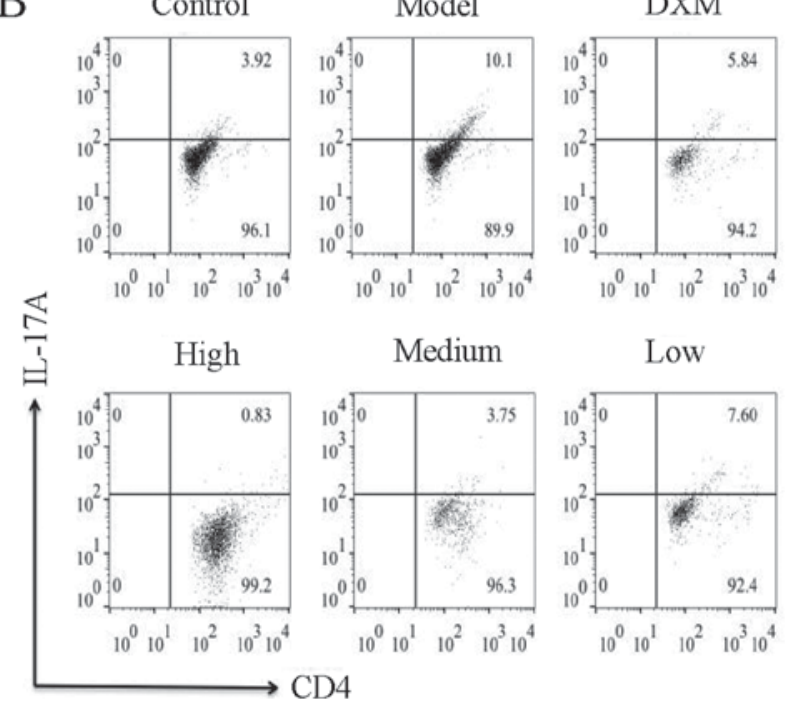

DXM

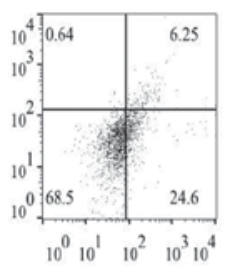

Low

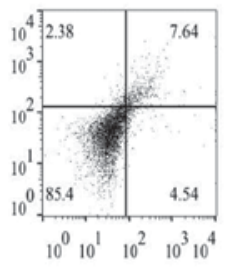

XM
D

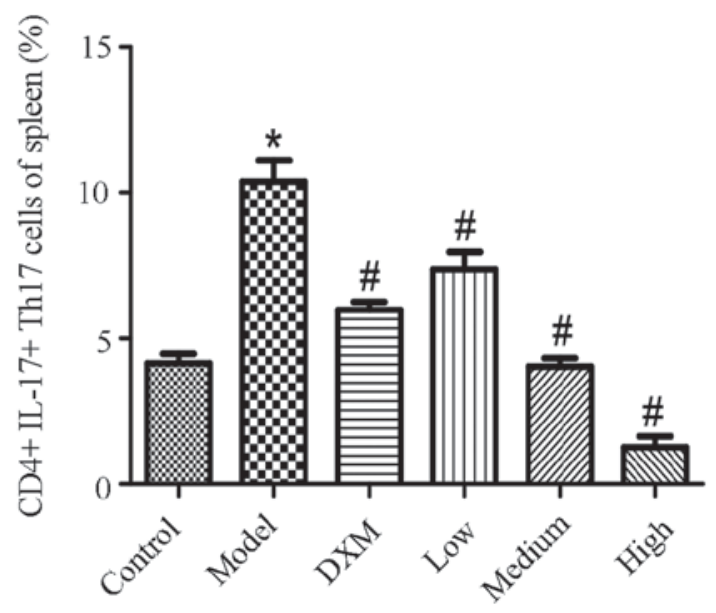

C

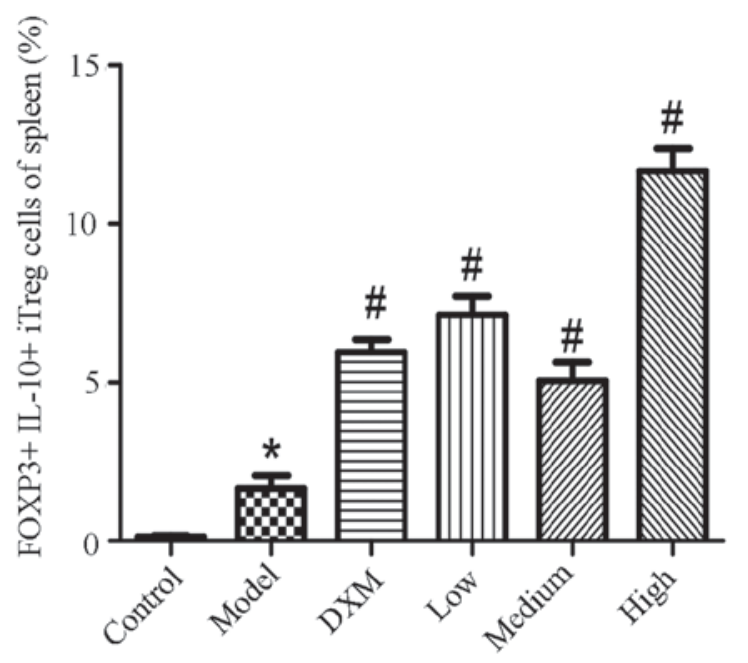

)

Figure 7. Trans-anethole regulates Treg and Th17 cells cytokines expression in the spleen tissue of mice. Flow cytometry of (A) Foxp3/IL-10 and (B) IL-17A/CD4, and (C and D) the quantification. (A) and (C) Treg cell cytokines expression altered in each group's spleen tissue. (B) and (D) Th17 cell cytokines expression altered in each group's spleen tissue. Statistics were displayed as the mean \pm standard deviation $(n=6)$. "P<0.05 vs. control; ${ }^{\text {" }} \mathrm{P}<0.05$ vs. model. IL, interleukin; DXM, Dexamethasone; Treg, T regulatory cells; Th17, T helper 17 cell; Foxp3, forkhead hox P3; CD4, cluster of differentiation.

cells, such as B cells and macrophages, and (2) to produce relevant cytokines, such as IL-10 $(38,39)$. In our study, we reported a decrease of Th17 cells and an increase of Tregs in the spleen tissue of the bronchitis model induced by LPS. The above results demonstrate that the change in ratio of Th17/Tregs may influence the pathogenesis of ALI caused by LPS. Tregs are a subdivision of $\mathrm{CD}^{+} \mathrm{T}$ cells that are important in maintaining self-tolerance to prevent autoimmunity, control chronic inflammatory diseases and to regulate lymphocytes (40). During the investigation of lymphocyte function in ALI caused by LPS, the study demonstrated that decreasing numbers of Tregs in mice with ALI decreased their capacity for recovery. This can be explained by a deficiency in the number of Tregs changing into lymphocytes that caused elevation of pro-inflammatory cytokines in the alveolar cavity and enhanced neutrophil apoptosis. This was confirmed in the present study as after treatment, Treg cells were obviously increased in the spleen tissue. Furthermore our study results are consistent with a recent study, which 
in modern pharmacology to have potential as a treatment of inflammatory diseases. In the current study, we demonstrated that trans-anethole, the main component of fennel, effectively alleviated airway inflammatory in ALI mice. The airway inflammation was alleviated via regulatory responses by Th17 and Tregs. The anti-inflammatory function of fennel has not been fully explained by molecular biology but the anti-inflammatory function of trans-anethole may be related to the reaction of Th17 and Tregs. Therefore, fennel may have potential as an effective treatment against inflammatory conditions.

\section{Acknowledgements}

Not applicable.

\section{Funding}

The present study was supported by the Projects from Administration Bureau of Traditional Chinese Medicine and Ethnic Group Medicine of Xinjiang Uyghur Autonomous Region (grant nos. Q2015-01-10 and 2017-01-10), Key Research and Development Program of Shandong Province (grant no. 2016ZDJS07A21) and the Program for Changjiang Scholars and Innovative Research Team in University (grant no. IRT_14R41).

\section{Availability of data and materials}

The datasets used or analysed during the current study are available from the corresponding author on reasonable request.

\section{Authors' contributions}

SZ implemented the hematoxylin and eosin staining, analyzed the data and wrote the manuscript. $\mathrm{CH}$ and LA executed the Th17/Treg examination in spleen tissue. QY and ID conducted reverse transcription-quantitative polymerase chain reaction. XC and SD counted the number of inflammatory cells. $\mathrm{XH}$ conceived the experiments and obtained the funding for this project. All authors read and agreed to the final manuscript.

\section{Ethics approval and consent to participate}

The present study was approved by the Animal Ethics Committee Of Laboratory Animal Care of Tianjin University of Traditional Chinese Medicine and was performed according to the guidelines of the Tianjin University of Traditional Chinese Medicine.

\section{Consent for publication}

Not applicable.

\section{Competing interests}

The authors declare that they have no competing interests.

\section{References}

1. Badgujar SB, Patel VV and Bandivdekar AH: Foeniculum vulgare Mill: A review of its botany, phytochemistry, pharmacology, contemporary application, and toxicology. Biomed Res Int 2014: 842674, 2014
2. Garg C, Khan SA, Ansari SH, Suman A and Garg M: Chemical composition, therapeutic potential and perspectives of Foeniculum vulgare. Pharmacogn Rev 3: 346-352, 2009.

3. Muckensturm B, Foechterlen D, Reduron JP, Danton P and Hildenbrand $\mathrm{M}$ : Phytochemical and chemotaxonomic studies of Foeniculum vulgare. Biochem Syst Ecol 25: 353-358, 1997.

4. Ostad SN, Soodi M, Shariffzadeh M, Khorshidi N and Marzban H: The effect of fennel essential oil on uterine contraction as a model for dysmenorrhea, pharmacology and toxicology study. J Ethnopharmacol 76: 299-304, 2001.

5. Malini T, Vanithakumari G, Megala N, Anusya S, Devi K and Elango V: Effect of Foeniculum vulgare Mill. seed extract on the genital organs of male and female rats. Indian J Physiol Pharmacol 29: 21-26, 1985.

6. Ghodsi Z and Asltoghiri M: The effect of fennel on pain quality, symptoms and menstrual duration in primary dysmenorrhea. J Pediatr Adol Gynec 27: 283-286, 2014.

7. Piras A, Falconieri D, Porcedda S, Marongiu B, Gonçalves MJ Cavaleiro C and Salgueiro L: Supercritical $\mathrm{CO}_{2}$ extraction of volatile oils from Sardinian Foeniculum vulgare ssp. vulgare (Apiaceae): Chemical composition and biological activity. Nat Prod Res 28: 1819-1825, 2014.

8. Damjanović B, Lepojević Ž, Živković V and Tolić A: Extraction of fennel (Foeniculum vulgare Mill.) seeds with supercritical $\mathrm{CO}_{2}$ : Comparison with hydrodistillation. Food Chem 92: 143-149, 2005.

9. Fang L, Qi M, Li T, Shao Q and Fu R: Headspace solvent microextraction-gas chromatography-mass spectrometry for the analysis of volatile compounds from Foeniculum vulgare Mill. J Pharm Biomed Anal 41: 791-797, 2006.

10. Pacifico S, Galasso S, Piccolella S, Kretschmer N, Pan SP, Nocera P, Lettieri A, Bauer R and Monaco P: Winter wild fennel leaves as a source of anti-inflammatory and antioxidant polyphenols. Arab J Chem (In press).

11. Kaur GJ and Arora DS: Antibacterial and phytochemical screening of Anethum graveolens, Foeniculum vulgare and Trachyspermum ammi. BMC Complement Altern Med 9: 30, 2009.

12. Kang P,Kim KY,Lee HS, Min SS and SeolGH: Anti-inflammatory effects of anethole in lipopolysaccharide-induced acute lung injury in mice. Life Sci 93: 955-961, 2013.

13. Namavar Jahromi B, Tartifizadeh A and Khabnadideh S: Comparison of fennel and mefenamic acid for the treatment of primary dysmenorrhea. Int J Gynaecol Obstet 80: 153-157, 2003.

14. Elsoud N, Ellaithy N, Elsaeed G, Wahby M, Khalil M, Morsy F and Shaffie N: Antidiabetic activities of Foeniculum vulgare Mill. essential oil in streptozotocin-induced diabetic rats. Maced J Med Sci 4: 139-146, 2011.

15. Koppula S and Kumar H: Foeniculum vulgare Mill (Umbelliferae) attenuates stress and improves memory in wister rats. Trop J Pharm Res 12: 553-558, 2013.

16. Ferguson ND, Fan E, Camporota L, Antonelli M, Anzueto A, Beale R, Brochard L, Brower R, Esteban A, Gattinoni L, et al: The Berlin definition of ARDS: An expanded rationale, justification, and supplementary material. Intensive Care Med 38: 1573-1582, 2012.

17. Matthay MA, Ware LB and Zimmerman GA: The acute respiratory distress syndrome. J Clin Invest 122: 2731-2740, 2012.

18. Mizgerd JP: Respiratory infection and the impact of pulmonary immunity on lung health and disease. Am J Respir Crit Care Med 186: 824-829, 2012.

19. Bhattacharya $J$ and Matthay MA: Regulation and repair of the alveolar-capillary barrier in acute lung injury. Ann Rev Physiol 75: 593-615, 2013.

20. Kim MR, Hong SW, Choi EB, Lee WH, Kim YS, Jeon SG, Jang MH, Gho YS and Kim YK: Staphylococcus aureus-derived extracellular vesicles induce neutrophilic pulmonary inflammation via both Th1 and Th17 cell responses. Allergy 67: 1271-1281, 2012.

21. Iwakura Y, Ishigame H, Saijo S and Nakae S: Functional specialization of interleukin-17 family members. Immunity 34: 149-162, 2011.

22. Risso K, Kumar G, Ticchioni M, Sanfiorenzo C, Dellamonica J, Guillouet-de Salvador F, Bernardin G, Marquette CH and Roger PM: Early infectious acute respiratory distress syndrome is characterized by activation and proliferation of alveolar T-cells. Eur J Clin Microbiol Infect Dis 34: 1111-1118, 2015.

23. Noack M and Miossec P: Th17 and regulatory T cell balance in autoimmune and inflammatory diseases. Autoimmun Rev 13: 668-677, 2014. 
24. Costantino CM, Baecher-Allan CM and Hafler DA: Human regulatory T cells and autoimmunity. Eur J Immunol 38: 921-924, 2008.

25. Guo Z, Wen Z, Qin A, Zhou Y, Liao Z, Liu Z, Liang Y, Ren T and $\mathrm{Xu} \mathrm{L}$ : Antisense oligonucleotide treatment enhances the recovery of acute lung injury through IL-10-secreting M2-like macrophage-induced expansion of CD4+ regulatory $\mathrm{T}$ cells J Immunol 190: 4337-4348, 2013.

26. Mills KH: Regulatory T cells: Friend or foe in immunity to infection? Nat Rev Immunol 4: 841-855, 2004.

27. Miossec P, Korn T and Kuchroo VK: Interleukin-17 and type 17 helper T cells. New Engl J Med 361: 888-898, 2009.

28. Nistala K, Moncrieffe H, Newton KR, Varsani H, Hunter P and Wedderburn LR: Interleukin-17-producing T cells are enriched in the joints of children with arthritis, but have a reciprocal relationship to regulatory $\mathrm{T}$ cell numbers. Arthritis Rheum 58: 875-887, 2008.

29. Tzartos JS, Friese MA, Craner MJ, Palace J, Newcombe J, Esiri MM and Fugger L: Interleukin-17 production in central nervous system-infiltrating $\mathrm{T}$ cells and glial cells is associated with active disease in multiple sclerosis. Am J Pathol 172: 146-155, 2008

30. Hirota K, Yoshitomi H, Hashimoto M, Maeda S, Teradaira S, Sugimoto N, Yamaguchi T, Nomura T, Ito H, Nakamura T, et al: Preferential recruitment of CCR6-expressing Th17 cells to inflamed joints via CCL20 in rheumatoid arthritis and its animal model. J Exp Med 204: 2803-2812, 2007.

31. Halwani R, Al-Muhsen S and Hamid Q: T helper 17 cells in airway diseases: From laboratory bench to bedside. Chest 143: 494-501, 2013.

32. Hartigan-O'Connor DJ, Hirao LA, McCune JM and Dandekar S: Th17 cells and regulatory T cells in elite control over HIV and SIV. Curr Opin HIV AIDS 6: 221-227, 2011.

33. You QH, Zhang D, Niu CC, Zhu ZM, Wang N, Yue Y and Sun GY: Expression of IL-17A and IL-17F in lipopolysaccharide-induced acute lung injury and the counteraction of anisodamine or methylprednisolone. Cytokine 66: 78-86, 2014.
34. Li C, Yang P, Sun Y, Li T, Wang C, Wang Z, Zou Z, Yan Y, Wang W, Wang C, et al: IL-17 response mediates acute lung injury induced by the 2009 pandemic influenza A (H1N1) virus. Cell Res 22: 528-538, 2012.

35. Fedele G, Spensieri F, Palazzo R, Nasso M, Cheung GY, Coote JG and Ausiello CM: Bordetella pertussis commits human dendritic cells to promote a Th1/Th17 response through the activity of adenylate cyclase toxin and MAPK-pathways. PLoS One 5: e8734, 2010.

36. Liu J, Zhang PS, Yu Q, Liu L, Yang Y, Guo FM and Qiu HB: Losartan inhibits conventional dendritic cell maturation and Thl and Th17 polarization responses: Novel mechanisms of preventive effects on lipopolysaccharide-induced acute lung injury. Int J Mol Med 29: 269-276, 2012.

37. Choi EM and Hwang JK: Antiinflammatory, analgesic and antioxidant activities of the fruit of Foeniculum vulgare. Fitoterapia 75: 557-565, 2004.

38. Taylor A, Verhagen J, Blaser K, Akdis M and Akdis CA Mechanisms of immune suppression by interleukin-10 and transforming growth factor-beta: The role of T regulatory cells. Immunology 117: 433-442, 2006.

39. De M, De AK, Sen P and Banerjee AB: Antimicrobial properties of star anise (Illicium verum Hook f). Phytother Res 16: 94-95, 2002.

40. Wang H, Peng W, Weng Y, Ying H, Li H, Xia D and Yu W: Imbalance of Th17/Treg cells in mice with chronic cigarette smoke exposure. Int Immunopharmacol 14: 504-512, 2012.

41. Scheffold A, Murphy KM and Höfer T: Competition for cytokines: T(reg) cells take all. Nat Immunol 8: 1285-1287, 2007.

This work is licensed under a Creative Commons Attribution-NonCommercial-NoDerivatives 4.0 International (CC BY-NC-ND 4.0) License. 